\title{
A Rule-Based Approach for the Recognition of Similarities and Differences in the Integration of Structural Karlstad Enterprise Modeling Schemata
}

\author{
Peter Bellström \\ Department of Information Systems, Karlstad University, 65188 Karlstad, Sweden \\ Peter.Bellstrom@kau.se
}

\begin{abstract}
In this paper, we address the recognition of similarities and differences in schema integration while applying the notation in the Karlstad Enterprise Modeling approach. In doing so, we describe and present a set of "if -then" rules. In these rules, we make use of both concept name comparison and comparison of concept neighborhoods (their surroundings). Following the classification of schema matching approaches given by [26], the rules are classified as a composite schema-based matching approach. The rules should first of all be viewed as a step towards a semi-automatic method for the recognition of similarities and differences in the integration of structural Karlstad Enterprise Modeling schemata. By applying the proposed rules, several problems, such as homonyms and synonyms, might be recognized that otherwise could pass unnoticed.
\end{abstract}

Keywords: Schema Integration, Recognition of Similarities and Differences, Karlstad Enterprise Modeling Approach.

\section{Introduction}

In the information systems development process, we often deal with requirements that are gathered from various sources. These requirements are often represented in the form of structural and behavioral schemata. The structural part represents both what should be stored in the database and what data the information system needs for processing the functionality it should provide. On the other hand, behavioral schemata represent what functionality the future information system should provide. In this paper, we delimit the discussion regarding the recognition of similarities and differences to structural schemata, also known as static schemata. In doing so, we focus on schema integration in conceptual database design. One of the most quoted definitions of schema integration is given by [1] where the authors define schema integration as "the activity of integrating the schemas of existing or proposed databases into a global, unified schema." (p. 323). Schema integration means that the source schemata are prepared, compared, modified and finally merged into one global schema representing parts of, or a whole, database on a conceptual level. In schema integration, it is important to maintain the vocabulary used in the source schemata otherwise semantic loss might occur [5]. Semantic loss is a problem that not only 
causes interpretation problems but also causes problems related to integration transparency.

In this paper, we present and describe an approach for the recognition of similarities and differences in schema integration. The approach is comprised of a set of rules starting with concept name comparison followed by concept neighborhood comparison. Following the classification of schema matching approaches given by [26], the rules are classified as a composite schema-based matching approach. The rules should first of all be viewed as a step towards a semi-automatic method for the recognition of similarities and differences in the integration of structural Karlstad Enterprise Modeling schemata. By applying the proposed rules, several problems, such as homonyms and synonyms, might be recognized that otherwise could pass unnoticed.

The paper is structured as follows: in section two, we address some examples of related work and distinguish it from our own rule approach. In section three, we present the Karlstad Enterprise Modeling approach with focus on the structural aspects. In section four, we present the main contributions given in this paper, namely the rules for the recognition of similarities and differences in the integration of structural Enterprise Modeling schemata. Finally, the paper closes with a summary together with conclusions and some proposals for future research.

\section{Related Work}

One way to classify earlier work in the domain of schema integration is to divide it into three parts [4] as follows:

- Manual approaches to schema integration

- Formal approaches to schema integration

- Semi-automatic approaches to schema integration

Manual means that all tasks, such as recognizing similarities and differences, are done manually. Formal means that some type of formal language is used and semiautomatic means that at least one computer application is used. In this section, we focus on the semi-automatic approaches, since the work presented in this paper should be viewed as a first step towards a semi-automatic method for the recognition of similarities and differences in the integration of structural Karlstad Enterprise Modeling schemata.

In [26] the authors present an overview of approaches to automatic schema matching. The authors distinguish between schema-based and instance-based matching, where schema-based matching can be performed for both the concept and its neighborhood. Further, schema based matching might be linguistic or constraintbased. The work presented in this paper is classified as a composite schema-based approach since first we compare concept names followed by a comparison of concept neighborhoods.

In [21] the authors present a method for structural conflict resolution while applying the Entity-Relationship modeling language [11]. Even though the title of the paper focuses on conflict resolution - resolving similarities and differences between two source schemata - the authors still address recognition. In doing so, the authors 
adopt an assertion-based method. The authors state that a "declarative statement asserting that a modeling construct [...] in one schema is somehow related to a modeling construct in another schema is called an inter-schema correspondence assertion or integration assertion for short." (p. 231). Since the authors use the EntityRelationship modeling language in their method, they focus on a level closer to implementation than the work presented in this paper.

In [28] the authors present a method for solving structural conflicts while applying a modified version of the Entity-Relationship modeling language called EntityRelationship for complex objects or ERC+ for short. In their approach, the authors point out that conflict resolution is performed without modification of the source schemata. The work presented in [28] focuses on ERC+, a modified version of the Entity-Relationship modeling language, and therefore also focuses on a level closer to implementation than the work presented in this paper.

In [30] the authors present an overview of their approach on how to integrate schemata on the pre-design level while applying the Klagenfurt Conceptual Predesign Model. A Klagenfurt Conceptual Pre-design Model schema should be viewed as an intermediate schema placed between natural language and more complex conceptual schemata designed with a more traditional modeling language, such as the Unified Modeling Language [24]. The work presented in [30] is in line with the work presented in this paper, meaning integration is done on a high level of abstraction and early in the process of information systems development.

In [19] the authors address schematic discrepancy in the integration of EntityRelationship schemata. According to the authors, schematic discrepancy is a problem that arises if "the same information is modeled as data in one database, but metadata in another." (p. 245). Schematic discrepancy is also a problem that might occur while applying the Karlstad Enterprise Modeling approach, since in our approach we have a symbol for instance-of (see Fig. 1). In other words, by applying the Karlstad Enterprise Modeling approach, it is possible to show that a concept in one schema is an instance-of a concept in another schema. Applying the instance-of dependency in conceptual database design might indicate several problems in schema integration, such as homonyms, that otherwise could pass unnoticed [3]. In [19] the authors adopt the Entity-Relationship modeling language, which again indicates that the authors are focusing on a level closer to implementation than the work presented in this paper.

Finally, it should be noted that in previous research, the Entity-Relationship modeling language, or some extension of it, has dominated schema integration research since the late 1980's [27], which is also reflected in this section.

\section{The Karlstad Enterprise Modeling Approach}

The Karlstad Enterprise Modeling (EM) approach refers to a modeling approach developed to address the pragmatic, the semantic and the syntactic aspects of an information system. The pragmatic aspects of EM are represented by a set of pragmatic dependencies (connections/links) used to illustrate goals, problems, and opportunities together with positive and negative influences [17].

The semantic aspects of EM are represented as both a set of static dependencies (see Fig. 1) and a set of dynamic dependencies. Furthermore, the dynamic dependencies are 
divided into state and communication dependencies. State dependencies are used to illustrate state changes together with conditions (both pre- and post) for actions. Communication dependencies are used to illustrate relations between actors, their actions and communication flows [16]. By applying the semantic aspects of EM, it is also possible to illustrate and represent system analysis patterns such as sequence, synchronization, iteration, selection and search [18] and to integrate static and dynamic aspects into one schema.

The syntactic aspects of EM are represented by a set of syntactic elements representing databases, database management systems, software components and human components. Syntactic aspects are also viewed as CASE-tool dependent and therefore the elements that are needed can be added on demand, meaning the list is not exhaustive [16].

One way to describe EM is as a generalization and extension of system analysis and design [16]. In [29] the author instead focuses on business processes and describes Enterprise Modeling as an approach that deals with modeling and integration of business processes.

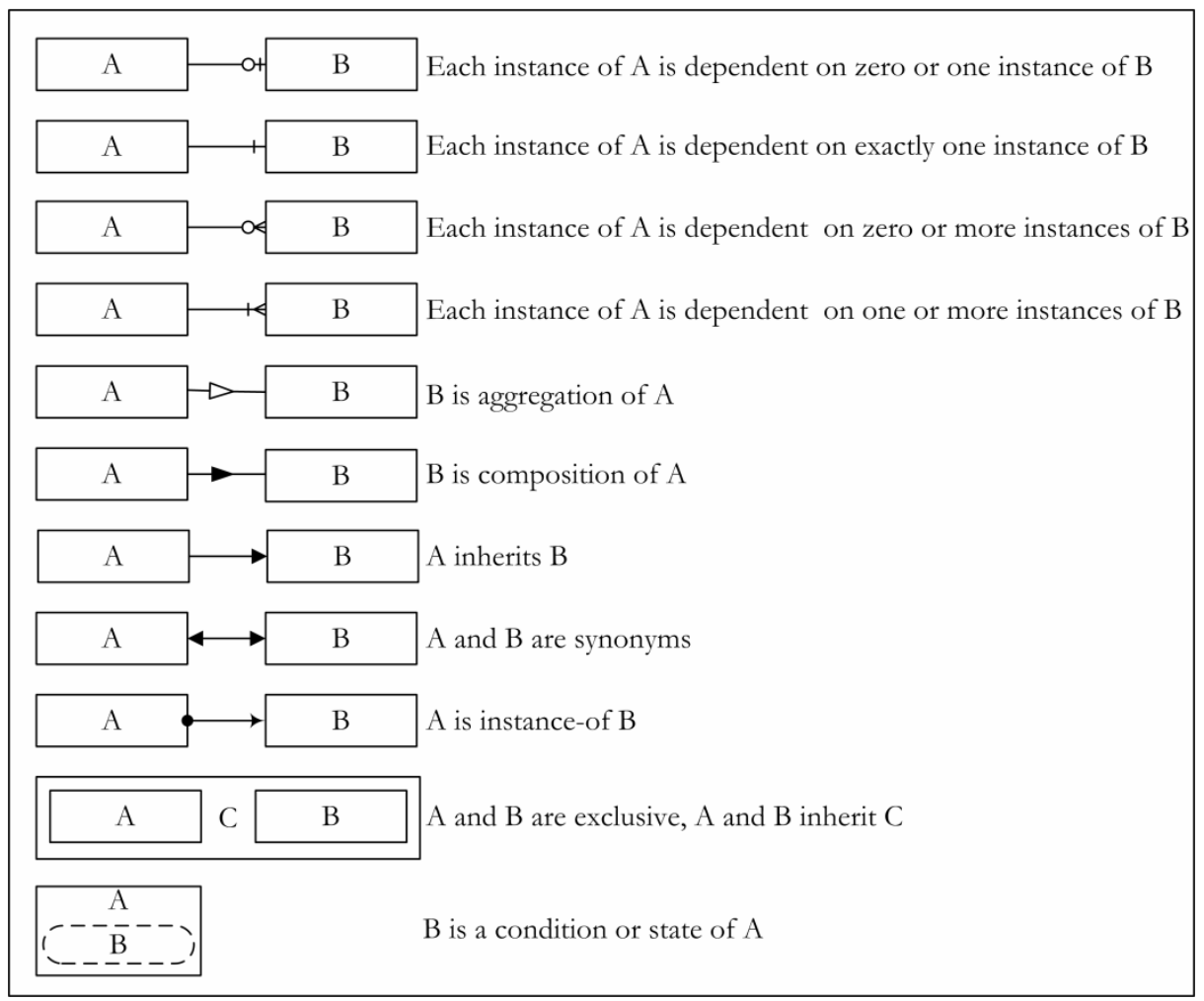

Fig. 1. Representation of static dependencies, structural aspects, in EM (adapted and modified from [16]) 
The structural aspects of EM (Fig. 1) show that boxes represent concepts and lines between concepts represent dependencies (connections/links). The structural dependencies illustrate what type of relation the concepts have to other concepts. For instance, one concept might be a specialization of another concept and one concept might be an instance-of another concept. It is important to point out that the only primitive, amongst the structural primitives, that is given a name is a concept. This also influences how similarities and differences between two EM schemata might be recognized. Compare this with the Entity-Relationship modeling language which names and distinct between entities, relationships and attributes.

Finally, while applying EM, we do not distinguish between classes and attributes but instead put focus on concepts and dependencies between them. This means that in our approach, we focus on content (what) and not on how the database is going to be implemented. This also means that our approach focuses on issues on a higher level of abstraction compared with traditional modeling languages such as the EntityRelationship modeling language [11] and the Unified Modeling Language [24].

\section{Recognition of Similarities and Differences in Schema Integration}

Several approaches and methods for the integration of structural schemata have been proposed during the years. In [1] the authors conclude that an integration method is comprised of, or at least a mixture of, the following four phases: pre-integration, comparison of the schemata, conforming the schemata, and merging and restructuring the schemata.

In pre-integration, each single source schema is translated into a canonical modeling language, checked for conflicts and inconsistencies (e.g. intra-schema similarities and differences) and the integration strategy is selected [27]. In comparison of the schemata the schemata are compared aiming to recognize name conflicts, structural conflicts and inter-schema properties [20] (e.g. inter-schema similarities and differences). In conforming the schemata, the similarities and differences recognized in the former phase are resolved. In the fourth and last phase, merging and restructuring the schemata, the schemata are first merged and later on also restructured.

Integration of structural schemata is important since there are often several users, or user groups, producing several schemata representing the same or different parts of a database. Several overlaps often exist between the produced schemata and therefore we need to integrate them into one schema. The method of first designing and later integrating conceptual schemata has been pointed out as important by many researchers. For instance [25] points out that local conceptual schemata preserve and highlight differences in how different users view their organization while a global conceptual schema may instead mask these. In [23] the authors mention that local conceptual schemata may not only prevent premature design decisions but also ensure that all local conceptual schemata are taken into account. Schema integration has also been mentioned as an effective - perhaps the most effective - technique for developing [14][15] and managing [22] large database schemata. Finally, as argued in [12] using a semantic model during conceptual design also simplifies schema integration. 
In this paper we put focus on the second phase in the integration method, comparison of the schemata, meaning we address how to recognize similarities and differences in the integration of structural Karlstad Enterprise Modeling schemata.

Comparison of the schemata has been described as not only important [27] but also as the most difficult phase in schema integration [13][21] and therefore needs more attention.

Our approach starts with comparison of concept names used in two structural schemata. The number of compared schemata is two since binary integration [1][2] is always assumed. If comparison of schema names yields either 'equivalent' or 'similar' the method continues with comparison of concept neighbors. This means comparing the concepts and dependencies directly connected to the compared concepts where a name match has been recognized [3][8]. The result of the proposed method might yield that either the compared concepts are 'equivalent', 'similar' or 'different'. However, this distinction is seldom easy to distinguish and therefore the following "if then" rules are proposed for equivalent concept names (E1-E7) and similar concept names (S1-S4) (see also [6]).

Before describing the rules, it should be noted that it is possible to complement the presented rule-based approach with not only a name comparison based on ontology but also a name comparison based on taxonomy [10].

It is also possible to complement the neighborhood comparison with several influence factors such as polysemy count, concept valency and domain weight. These influence factors could be used to relieve and optimize an implementation of our approach, meaning the influence factors could be used to decide if neighborhood comparison is even necessary.

A polysemy count gives the number of meanings a word has in a specific language, concept valency gives the number of parameters a word needs for getting its full meaning and a domain weight can be manually given to concepts by a domain expert.

However, it should be noted that whenever ambiguity exists it is recommended to continue with a neighborhood comparison [10].

\subsection{Rules for Equivalent Concept Names}

In rules E1-E7, name comparison always yields equivalent, meaning the compared concept names (labels) are the same. The rules for equivalent concept names are:

Rule E1: If comparison of concept names yields equivalent, and comparison of concept neighborhoods yields equivalent then equivalent concepts might be recognized.

Rule E2: If comparison of concept names yields equivalent and comparison of concept neighborhoods yields different then homonyms might be recognized between the two concept names in the source schemata. Homonyms occur if one name is used for several concepts with different meanings. For instance using concept name Name in the context of Customer (schema one) and in the context of Product (schema two). Fig. 2 illustrates the recognition of homonyms by applying Rule E2. In Fig. 2, comparison of concept names yields equivalent for the concept named Name. However, comparison of concept neighborhoods yields no match, which could indicate homonyms between the two source schemata. 


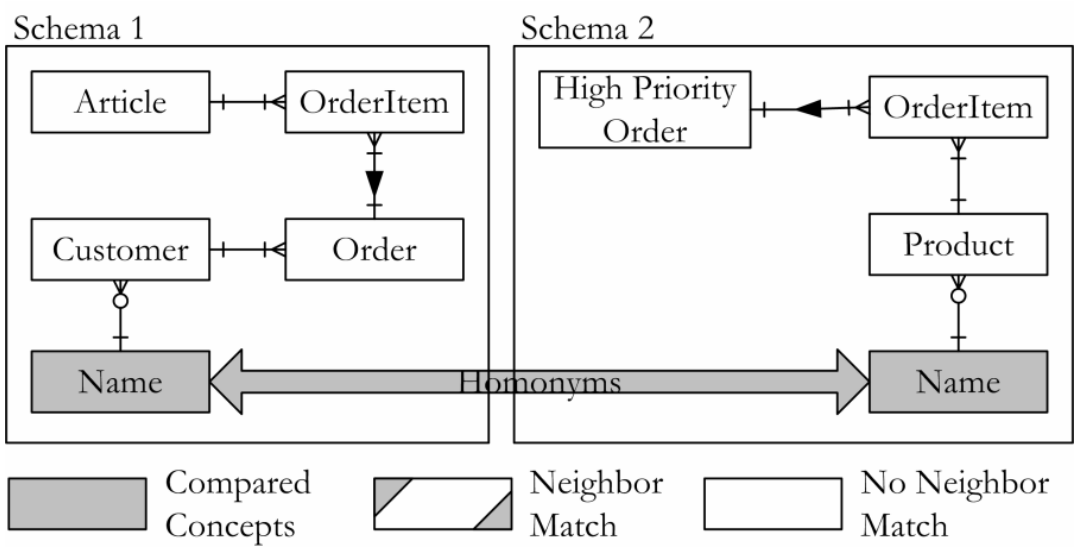

Fig. 2. Recognition of homonyms based on Rule E2

Rule E3: If comparison of concept names yields equivalent and comparison of concept neighborhoods yields similar, meaning one concept in each source schemata is named different, then synonyms might be recognized between the two concept names. Synonyms occur if several names are used for one concept with the same meaning. For instance using concept name Article in schema one and Product in schema two. Fig. 3 illustrates recognition of synonyms by applying Rule E3. In Fig. 3, comparison of concept names yields equivalent for the concept named OrderItem. However, comparison of concept neighborhoods yields similar meaning; one concept in each schema is named differently, which could indicate synonyms between the two source schemata.

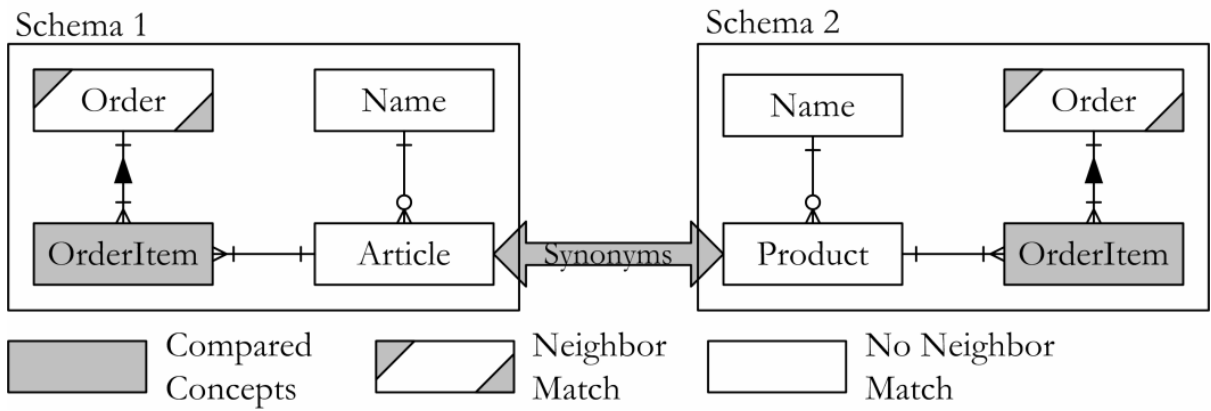

Fig. 3. Recognition of synonyms based on Rule E3

Rule E4: If comparison of concept names yields equivalent and comparison of concept neighborhoods yields similar, meaning one concept name in one of the source schema is a composite of a concept name in the other source schema with a following addition, and cardinality indicates "one-to-one", then an association dependency between the two concept names might be recognized. An association between two concepts means that they have a trivial dependency (link/connection) to each other and the cardinality is specified. For instance, using Customer in schema one and 
Customer Number in schema two. Fig. 4 illustrates recognition of an association dependency between Customer in Schema 1 and Customer Number in Schema 2 by applying Rule E4. In Fig. 4, comparison of concept names yields equivalent for the concept named Name. However, comparison of concept neighborhood yields similar meaning; Customer Number is a composite of Customer in schema 1 with the following addition Number and cardinality indicates uniqueness ("one-to-one").

Schema $1 \quad$ Schema 2

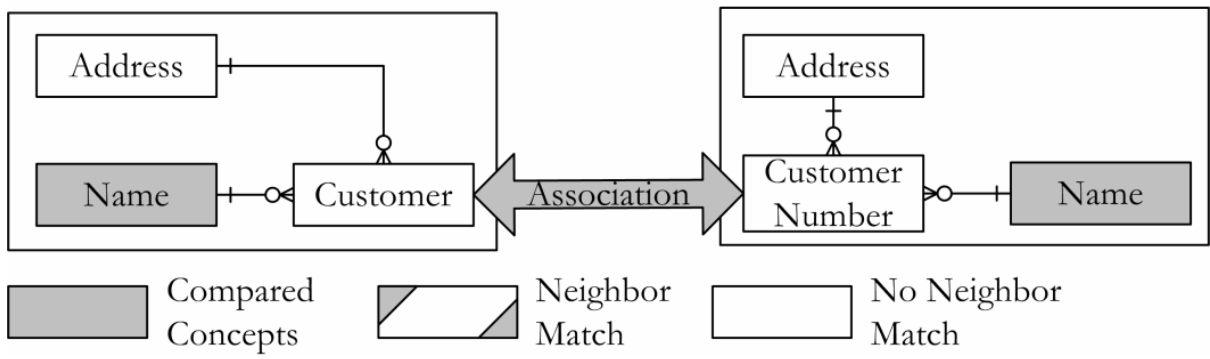

Fig. 4. Recognition of association based on Rule E4

Rule E5: If comparison of concept names yields equivalent and comparison of concept neighborhoods yields similar, meaning one concept name in one of the source schemata is a composite of a concept name in the other source schema, with a prior addition, then a hypernym-hyponym dependency might be recognized between the two concept names. A hypernym-hyponym dependency occurs if one concept is recognized as a specialization of another concept, which at the same time is recognized as a generalization of the other concept. A hypernym-hyponym dependency is also known as inheritance (is-a) and as generalization-specialization (see Fig.1). For instance, using High Priority Order (hyponym) in schema one and Order (hypernym) in schema two. In Fig. 5, comparison of concept names yields equivalent for the concept named OrderItem. However, comparison of concept neighborhood yields similar neighbor match, which could indicate a hypernymhyponym dependency between the two source schemata.

Schema $1 \quad$ Schema 2

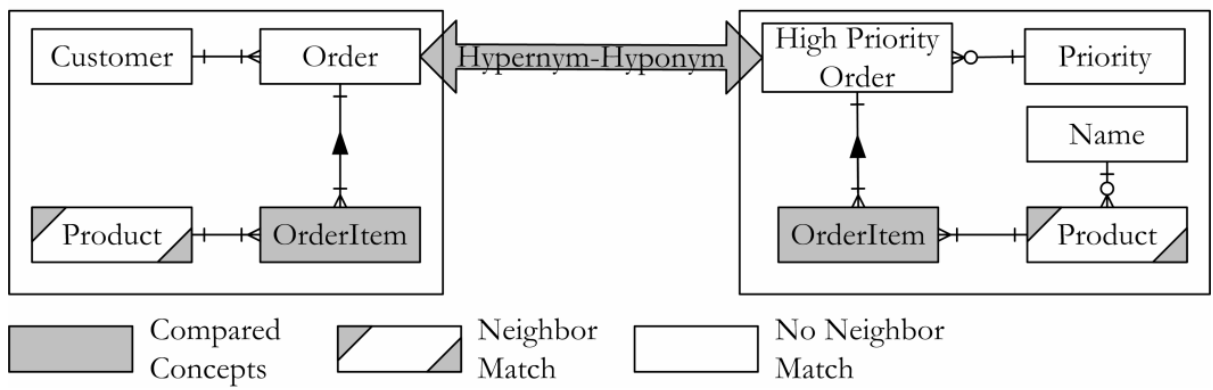

Fig. 5. Recognition of a hypernym-hyponym dependency based on Rule E5 
Rule E6: If comparison of concept names yields equivalent and comparison of concept neighborhoods yields similar, meaning one concept name in one of the source schema is a composite of a concept name in the other source schema with a following addition and cardinality indicates uniqueness with "many", then a holonym-meronym dependency (composition) between the two concept names might be recognized. A holonym-meronym dependency occurs if once concept is recognized as a part of another concept, which at the same time is recognized as composed (see Rule E6) or aggregated (see Rule E7) of the other concept(s). The holonym-meronym dependency is also known as aggregation and composition (see Fig. 1). For instance, using Order in schema one and OrderItem in schema two. In Fig. 6, comparison of concept names yields equivalent for the concept named OrderItem. However, comparison of concept neighborhood yields similar neighbor match, which could indicate a holonymmeronym dependency between the two source schemata.

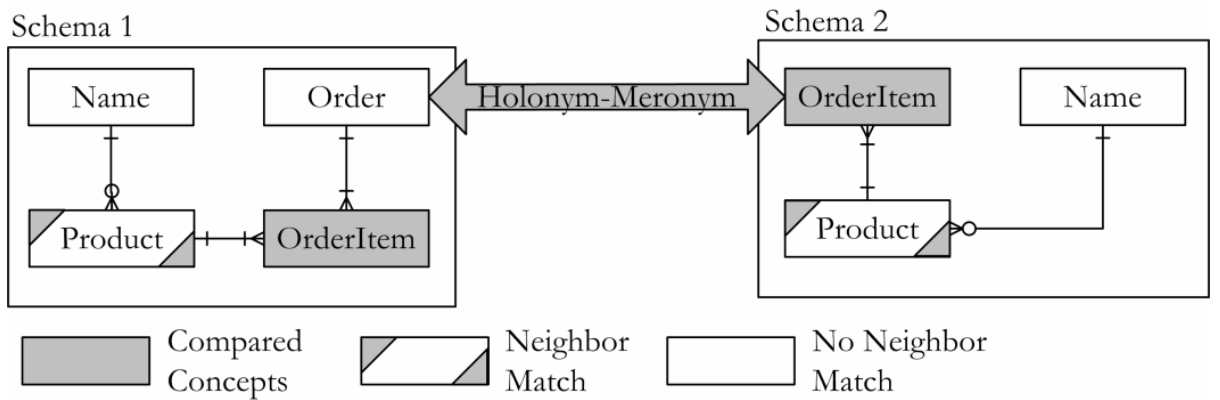

Fig. 6. Recognition of a holonym-meronym dependency based on Rule E6

Rule E7: If comparison of concept names yields equivalent and comparison of concept neighborhoods yields similar, meaning one concept name in one of the source schema is a composite of a concept name in the other source schema with a following addition and the cardinality between the two concepts indicates "many" without uniqueness, then a holonym-meronym dependency (aggregation) between the two concept names might be recognized. For instance using Order in schema one and OrderLine in schema two.

A few issues need to be addressed in the presented rules. Depending on how the dependencies are used in the source schemata, rules four, five and six could indicate either sharper or looser dependencies. This means that rule E4 could indicate an association or a composition (part-of) dependency and rule E5 and E6 could indicate an association dependency. It should also be noted that in schema integration synonyms and homonyms are often called name conflicts [1] or semantic conflicts [27] and hypernym-hyponym and holonym-meronym dependencies as inter-schema properties [20]. An inter-schema property occurs if two source schemata have certain constraints in common [20] such as a hypernym-hyponym dependency and a holonym-meronym dependency. 


\subsection{Rules for Similar Concept Names}

In rules S1-S4, name comparison always yields similar, meaning the compared concept names (labels) are not exactly the same but very similar. Rules S1-S4 can be viewed as a refinement and extension of the two rules expressed and proposed in [10] stated as:

- Rule 1: If concept $\mathrm{A}$ is atomic and concept $\mathrm{AB}$ is a composite consisting of A followed by another word B then the concept AB 'belongs-to' concept A. Example: "Customer" and "Customer Name" $\rightarrow$ "Customer Name" 'belongs-to' "Customer".

- Rule 2: If concept A is atomic and concept BA is a composite consisting of a word B followed by word A then the concept BA 'is-a' concept A. Example: "Order" and "High Priority Order" $\rightarrow$ "High Priority Order" 'is-a' “Order”. (p. 112)

However, the rules expressed and proposed in [10] are proposed for any modeling language, meaning the rules are modeling language independent, while the rules described in this paper are adapted for the Karlstad Enterprise Modeling approach. The rules for similar concept names are:

Rule S1: If comparison of concept names yields similar, meaning one concept name and one concept name with a following addition to the first one, and comparison of concept neighborhoods yields similar or equivalent with a "one-to-one" in cardinality then an association dependency between the two concept names might be recognized. For instance using Customer in schema one and Customer Id in schema two.

Rule S2: If comparison of concept names yields similar, meaning one concept name and one concept name with a following addition to the first one, and comparison of concept neighborhoods yields similar or equivalent with uniqueness and "many" in cardinality then a holonym-meronym dependency (composition) between the two concept names might be recognized. For instance using Order in schema one and OrderItem in schema two.

Rule S3: If comparison of concept names yields similar, meaning one concept name and one concept name with a following addition to the first one, and comparison of concept neighborhoods yields similar or equivalent and the cardinality indicates "many" without uniqueness then a holonym-meronym dependency (aggregation) between the two concept names might be recognized. For instance using Order in schema one and OrderLine in schema two.

Rule S4: If comparison of concept names yields similar, meaning one concept name and one concept name with a prior addition to the first one, and comparison of concept neighborhoods yields similar or equivalent, then a hypernym-hyponym dependency might be recognized between the two concept names. For instance using High Priority Order (hyponym) in schema one and Order (hypernym) in schema two.

Occasionally it could be useful to continue with comparison of concept neighborhoods even if the comparison of concept names yields different (see also [10]). In doing so, synonyms that otherwise could pass unnoticed might be recognized (see Fig. 7). The result of a comparison of concept neighborhoods, with the prior 
result of different from comparison of concept names, could result in three cases. If different, then the concept names are different or if similar or equivalent then concept names could be synonyms. In line with the issues addressed in connection with rules E4-E6, rules S1-S3 could indicate either sharper or looser dependencies. In Fig. 7, two concept names yield equivalent (see Name and OrderItem). Based on these two matches, a neighborhood comparison might be conducted indicating that Article in Schema one and Product in Schema two are synonyms.

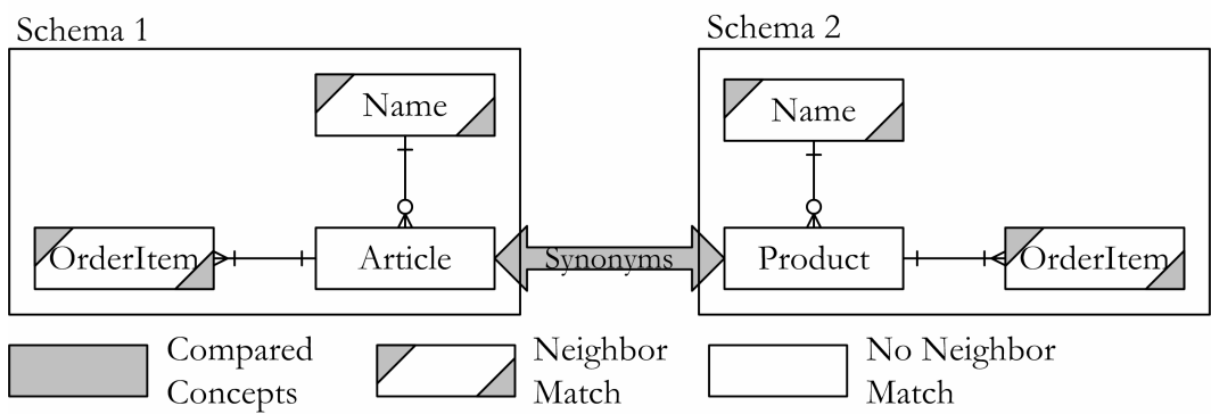

Fig. 7. Recognition of synonyms based on neighborhood comparison

\section{Summary and Conclusions}

In this paper we have described and illustrated a rule-based approach for the recognition of similarities and differences in the integration of structural Karlstad Enterprise modeling schemata. Stating the chosen modeling language and approach is very important since the chosen modeling language often influences not only how similarities and differences between two source schemata are recognized but also how similarities and differences between two source schemata are resolved in the integration process.

In the Karlstad Enterprise Modeling approach, structural and behavioral aspects are closely connected, they might even be integrated into one schema, and similar problems, such as homonyms and synonyms, might occur while integrating each type of schema (structural and behavioral). Therefore the work presented in this paper is also partially applicable to the integration of behavioral schemata. Integration of behavioral schemata is a topic that will be addressed in depth in future research. However, before integrating the behavioral schemata it is preferable to integrate the structural schemata. The motivation for this is that then several problems, such as homonyms and synonyms, will already have been resolved [7]. Additionally, the integrated structural schemata can be used as an information resource while integrating the behavioral schemata [9].

The presented and illustrated rule-based approach should first of all be viewed as a step towards a semi-automatic method for the recognition of similarities and differences in the integration of structural Karlstad Enterprise Modeling schemata. As shown in section four, by applying the proposed rules several problems, such as homonyms, synonyms, associations, hypernyms-hyponyms and holonyms-meronyms, might be recognized that otherwise could pass unnoticed. 
Future research will focus on developing a semi-automatic method for the integration of structural Karlstad Enterprise modeling schemata. In future research we will also address methods and approaches, both manual and semi-automatic, for the integration of behavioral Karlstad Enterprise modeling schemata.

\section{References}

1. Batini, C., Lenzerini, M., Navathe, S.B.: A Comparative Analysis of Methodologies for Database Schema Integration. ACM Computing Surveys 18(4), 323-364 (1986)

2. Batini, C., Ceri, S., Navathe, S.B.: Conceptual Database Design An Entity-Relatinship Approach. The Benjamin/Cummings Publishing Company, Redwood City (1992)

3. Bellström, P.: Using Enterprise Modeling for Identification and Resolution of Homonym Conflicts in View Integration. In: Vasilecas, O., Caplinskas, A., Wojtkowski, W., Wojtkowski, W.G., Zupančič, J., Wrycza, S. (eds.) Information Systems Development Advances in Theory, Practice, and Education, pp. 265-276. Springer, Heidelberg (2005)

4. Bellström, P.: View Integration in Conceptual Database Design Problems, Approaches and Solutions. Licentiate Thesis. Karlstad University Studies 2006:5 (2006)

5. Bellström, P.: On the Problem of Semantic Loss in View Integration. In: Barry, C., Conboy, K., Lang, M., Wojtkowski, G., Wojtkowski, W. (eds.) Information Systems Development Challenges in Practice, Theory, and Education, pp. 963-974. Springer Science, Heidelberg (2009)

6. Bellström, P.: Schema Integration - How to Integrate Static and Dynamic Database Schemata. Dissertation. Karlstad University Studies 2010:13 (2010)

7. Bellström, P., Jakobsson, L.: Towards a Generic and Integrated Enterprise Modeling Approach to Designing Databases and Software Components. In: Nilsson, A.G., Gustas, R., Wojtkowski, W., Wojtkowski, S.W., Wrycza, S., Zupancic, J. (eds.) Advances in Information Systems Development: Bridging the Gap between Academia and Industry, pp. 635-646. Springer Science, Heidelberg (2006)

8. Bellström, P., Vöhringer, J., Salbrechter, A.: Recognition and Resolution of Linguistic Conflicts: The Core to a Successful View and Schema Integration. In: Magyar, G., Knapp, G., Wojtkowski, W., Wojtkowski, W.G., Zupančič, J. (eds.) Advances in Information Systems Development: New Methods and Practice for the Networked Society, pp. 77-87. Springer Science, Heidelberg (2007)

9. Bellström, P., Vöhringer, J., Kop, C.: Guidelines for Modeling Language Independent Integration of Dynamic Schemata. In: Pahl, C. (ed.) Proceedings of the IASTED International Conference on Software Engineering, pp. 112-117. ACTA Press (2008)

10. Bellström, P., Vöhringer, J.: Towards the Automation of Modeling Language Independent Schema Integration. In: Kusiak, A., Lee, S.-G. (eds.) Proceedings International Conference on Information, Process, and Knowledge Management, pp. 110-115. IEEE Computer Society, Los Alamitos (2009)

11. Chen, P.: The Entity-Relationship Model - Toward a Unified View of Data. ACM Transactions on Database Systems 1(1), 9-36 (1976)

12. Ekenberg, L., Johannesson, P.: Conflictfreeness as a Basis for Schema Integration. In: Bhalla, S. (ed.) CISMOD 1995. LNCS, vol. 1006, pp. 1-13. Springer, Heidelberg (1995)

13. Ekenberg, L., Johannesson, P.: A Formal Basis for Dynamic Schema Integration. In: Thalheim, B. (ed.) Conceptual Modeling, ER 1996, pp. 211-226. Springer, Heidelberg (1996) 
14. Frank, H., Eder, J.: Integration of Behavioral Models. In: Proceedings of the ER 1997 Workshop on Behavioral Models and Design Transformations: Issues and Opportunities in Conceptual Modeling [Electronic] (1997),

http: / / osm7 . cs . byu . edu / ER97 / workshop4 / fe.html (04-08-2010)

15. Frank, H., Eder, J.: Integration of Statecharts. In: Proceedings of the 3rd IFCIS International Conference on Cooperative Information Systems, pp. 364-372. IEEE Computer Society, Los Alamitos (1998)

16. Gustas, R.: Gustiené. P.: Towards the Enterprise Engineering Approach for Information System Modelling Across Organisational and Technical Boundaries. In: Camp, O., Filipe, J., Hammoudi, S. \& Piattini, M. (eds.) Enterprise Information Systems V. pp. 204-215. Kluwer (2004)

17. Gustas, R., Gustiené, P.: Pragmatic-Driven Approach for Service-Oriented Analysis and Design. In: Johannesson, P., Söderström, E. (eds.) Information Systems Engineering: From Data Analysis to Process Networks, pp. 97-128. IGI Global (2008)

18. Gustas, R., Gustiené, P.: Service-Oriented Foundation and Analysis Patterns for Conceptual Modelling of Information Systems. In: Barry, C., Conboy, K., Lang, M., Wojtkowski, G., Wojtkowski, W. (eds.) Information Systems Development Challenges in Practice, Theory, and Education, pp. 249-265. Springer Science, Heidelberg (2009)

19. He, Q., Ling, T.W.: Resolving Schematic Discrepancy in the Integration of EntityRelationship Schemas. In: Atzeni, P., Chu, W., Lu, H., Zhou, S., Ling, T.W. (eds.) ER 2004. LNCS, vol. 3288, pp. 245-258. Springer, Heidelberg (2004)

20. Johannesson, P.: Schema Integration, Schema Translation, and Interoperability in Federated Information Systems. Dissertation. Stockholm: Department of Computer \& Systems Sciences, Stockholm University (1993)

21. Lee, M.L., Ling, T.W.: A Methodology for Structural Conflict Resolution in the Integration of Entity-Relationship Schemas. Knowledge and Information System 5(2), 225-247 (2003)

22. Mannino, M.V.: Database Design, Application Development, \& Administration. McGrawHill/Irwin, Irwin (2007)

23. Nuseibeh, B., Easterbrook, S., Russo, A.: Making Inconsistency Respectable in Software Development. The Journal of Systems and Software 58, 171-180 (2001)

24. Object Management Group: OMG Unified Modeling Language (OMG UML), Superstructure. [Electronics] (2009),

http: / / www . omg . org/spec/UML/2 . 2 / Superstructure/PDF / (25-01-2010)

25. Parsons, J.: Effects of Local Versus Global Schema Diagrams on Verification and Communication in Conceptual Data Modeling. Journal of Management Information Systems 19(3), 155-183 (2003)

26. Rahm, E., Bernstein, P.A.: A Survey of Approaches to Automatic Schema Matching. VLDB Journal 10, 334-350 (2001)

27. Song, W.: Schema Integration - Principles, Methods, and Applications. Dissertation. Stockholm: Department of Computer and Systems Sciences, Stockholm University (1995)

28. Spaccapietra, S., Parent, C.: View Integration: a Step Forward in Solving Structural Conflicts. IEEE Transactions on Knowledge and Data Engineering 6(2), 258-274 (1994)

29. Vernadat, F.B.: Enterprise Modeling and Integration: principles and applications. Chapman \& Hall, Boca Raton (1996)

30. Vöhringer, J., Mayr, H.C.: Integration of Schemas on the Pre-Design Level Using the KCPM-Approach. In: Nilsson, A.G., Gustas, R., Wojtkowski, W., Wojtkowski, W.G., Wrycza, S., Zupančič, J. (eds.) Advances in Information Systems Development: Bridging the Gap between Academia and Industry, pp. 623-634. Springer Science, Heidelberg (2006) 